\title{
Cell Culture Monitoring System Comprising Superoxide Sensors for Tumour Cell Analysis
}

\author{
H. Flamm $^{1}$, J. Kieninger ${ }^{1}$, K. Aravindalochanan ${ }^{1}$, G. A. Urban ${ }^{1}$ \\ E. Schleicher', A.M. Rostas', S. Weber ${ }^{2}$ \\ ${ }^{1}$ Albert-Ludwigs-Universität Freiburg - Department of Microsystems Engineering (IMTEK), Georges- \\ Köhler-Allee 103, 79110 Freiburg, Germany \\ ${ }^{2}$ Albert-Ludwigs-Universität Freiburg - Institut für Physikalische Chemie, Albertstraße 21, \\ 79104 Freiburg, Germany \\ Hubert.Flamm@imtek.uni-freiburg.de
}

\begin{abstract}
:
A sensor for the selective and sensitive measurement of reactive oxygen species (ROS) candidate superoxide was developed using direct electrochemical oxidation of superoxide radical on gold electrodes at low operation potential. Sensor performance regarding sensitivity and selectivity was investigated and optimised by integration of polymer based cut-off membranes. Sensor calibration was done utilising the artificial enzymatic based production of short-lived superoxide radicals by xanthine/xanthine oxidase system. The reaction kinetics of superoxide production was investigated with electrochemical, optical and magneto-spectroscopic measurement methods. Sensor design and fabrication process allow a full integration of superoxide sensor in existing multiparameter measurement platform SCCF for in vitro cell culture monitoring.
\end{abstract}

Key words: Superoxide anion radical, ROS, cell culture monitoring, electrochemical sensor, SCCF

\section{Introduction}

The detection of free radicals, especially superoxide anions, has been considered extensively in the past few years due to their tremendous role in neurodegenerative and cancer related diseases. Mostly electrochemical protein or enzyme based microsensors are used for in vitro as well as for in vivo sensing of superoxide. These sensors can show high sensitivity to superoxide radical but often longtime stability and selectivity is not achieved due to degradation effects or protein inactivation. The presented electrochemical superoxide microsensor system demonstrates the application of metal microelectrodes without the need of enzymes for extended operation time and good sensitivity, thus it is suitable for cell culture monitoring over longer period of time.

\section{Technology}

The superoxide sensing electrode is developed to be integrated in the technical monitoring platform Sensing Cell Culture Flask (SCCF) for in vitro cell research [1]. The platform utilises conventional tissue culture flask with integrated sensor chips at the bottom (see figure 1). Substrate material for the sensing chips is glass, thus allowing optical inspection of the cells during the complete culturing period. The SCCF comprises a complete electrochemical analysis system for multiplexed monitoring of different parameters in amperometric and potentiometric mode. Sensors for oxygen, nitric oxide, $\mathrm{pH}$ and temperature are implemented in the system, whereas nearly any type of electrochemical biosensor can be equipped [2]

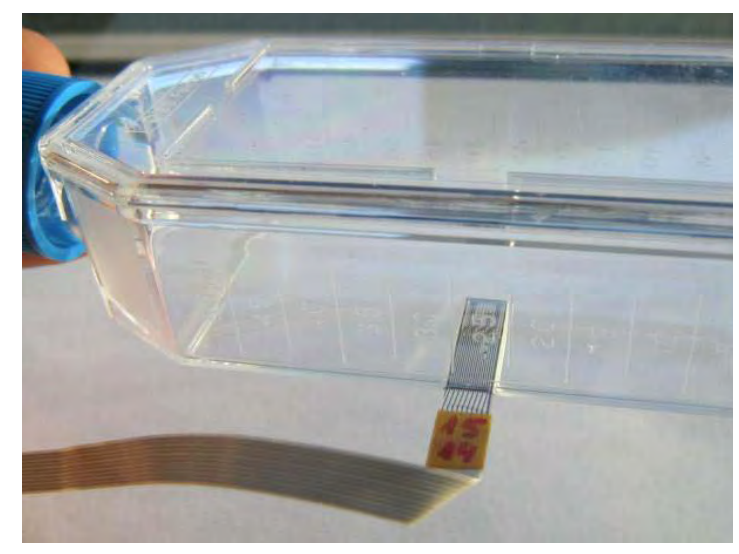

Figure 1: Sensing Cell Culture Flask (SCCF) with integrated sensor chip for pericellular in vitro measurement.

Superoxide sensing electrodes are based on micro-machined thin-film gold layers or galvanic electrodeposited gold as post process on SCCF 
chips. Electropolymerised phenylenediamine or plasmapolymer [3] was individually processed on blank gold electrodes to enhance the selectivity. All fabrication steps are process compatible with the other sensing electrodes on the SCCF chip.

\section{Superoxide generation}

Sensor characterisation and calibration of superoxide sensor was investigated with artificial enzymatic production of superoxide. The reaction of xanthine with xanthine oxidase produces uric acid and short-lived superoxide with reaction rate $\mathrm{k}_{1}$ according to [4]

$$
\text { Xanthine }+\mathrm{O}_{2}+\mathrm{H}_{2} \mathrm{O} \rightarrow \text { uric acid }+\mathrm{O}_{2}^{--}
$$

Superoxide molecules decompose spontaneously to hydrogen peroxide with reaction rate $\mathrm{k}_{2}$ according to equation

$$
2 \mathrm{O}_{2}^{--}+2 \mathrm{H}^{+} \rightarrow \mathrm{O}_{2}+\mathrm{H}_{2} \mathrm{O}_{2}
$$

Thus a steady-state concentration of superoxide molecules establishes after short time [5] with

$$
\left[\mathrm{O}_{2}^{--}\right]_{\mathrm{st}}=\sqrt{\frac{\mathrm{k}_{1}}{2 \mathrm{k}_{2}}[\mathrm{XOD}]}
$$

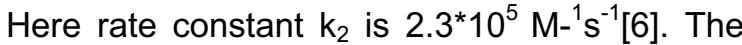
rate constant $\mathrm{k}_{1}$ was determined using cytochrome $C$ reduction assay with $\mathrm{k}_{1}=1.4 \mathrm{~s}^{-1}$. The formation of superoxide radical was also investigated by highly selective electron paramagnetic resonance spectroscopy (EPR) to determine the reaction yield of equation (1).

Figure 2 shows an EPR measurement with fixed XOD concentration and increased xanthine concentrations producing increasing amount of spin-trapped superoxide in a linear dependency.

These results suggest the suitability of the xanthine/xanthine oxidase system for the reliable artificial production of steady-state superoxide concentrations for sensor calibration and characterisation.

\section{Sensor characterisation}

Cyclic voltammetry studies on blank thin-film gold electrodes revealed a lower oxidation potential for superoxide than for the most

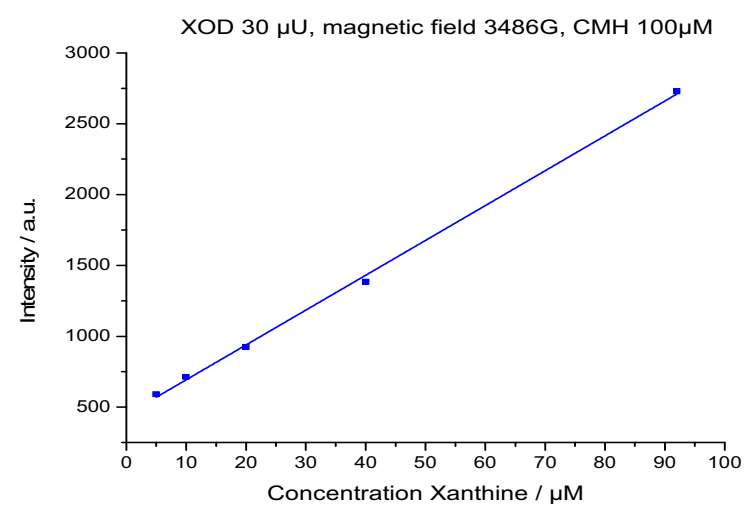

Figure 2: EPR signal peak height for different concentrations of $\mathrm{CMH}$ spin-trapped superoxide radicals.

relevant interfering substances hydrogen peroxide and uric acid. Covering the blank electrodes with thiol-containing linker molecule cysteine, used as peptide linker in conventional enzyme based superoxide sensors leads to a severe lowering in the oxidation potential of interferents at gold electrodes. Thus the direct oxidation of superoxide on blank electrodes is the preferred method for a selective measurement.

Superoxide production was done according to equation (1) with $100 \mu \mathrm{M}$ xanthine solution in phosphate buffered saline solution (100mM PBS $\mathrm{pH}=7.4$ ). Adding xanthine oxidase aliquots to the stirred solution leads to a stepwise increase in superoxide production and establishes a steady-state concentration. An amperometric measurement of increasing superoxide concentration at $+200 \mathrm{mV}$ vs. $\mathrm{Ag} / \mathrm{AgCl} 3 \mathrm{M} \mathrm{KCl}$ reference electrode is shown in figure 3 .

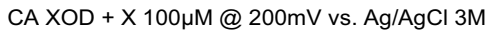

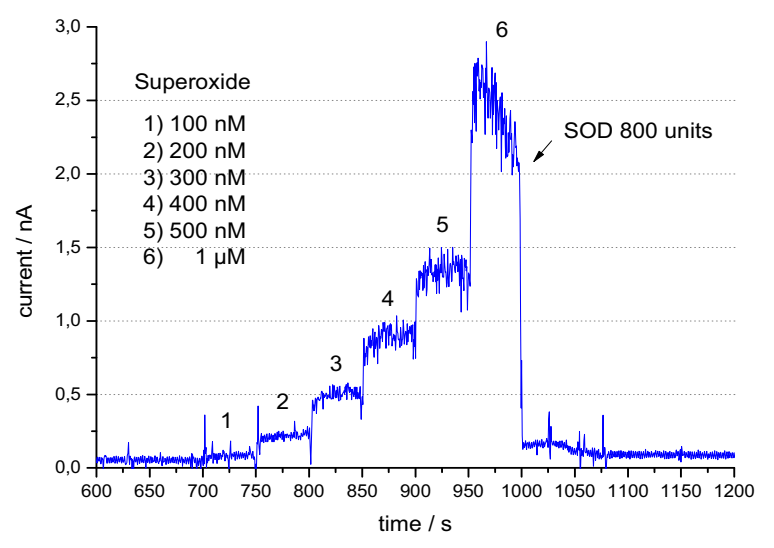

Figure 3: Chronoamperometric measurement of superoxide at blank gold electrode. Artificial production in $100 \mathrm{mM}$ PBS $\mathrm{pH} 7.4$ with $100 \mu \mathrm{M}$ xanthine by successive addition of xanthine oxidase. Arrow indicates superoxide scavenging by superoxide dismutase (SOD). 
After adding 400U/ml enzyme superoxide dismutase (SOD) the signal immediately drops to nearly baseline level indicating an effective scavenging of superoxide radicals by the highly selective enzyme. As baseline level is reached, no significant interferent oxidation can be observed. Figure 4 illustrates the signal height of steady-state superoxide concentration in comparison with the main interfering substances uric acid, hydrogen peroxide and ascorbic acid at the measurement potential. A linear dependency of superoxide oxidation current to the calculated superoxide concentration, according to equation (1), can be achieved.

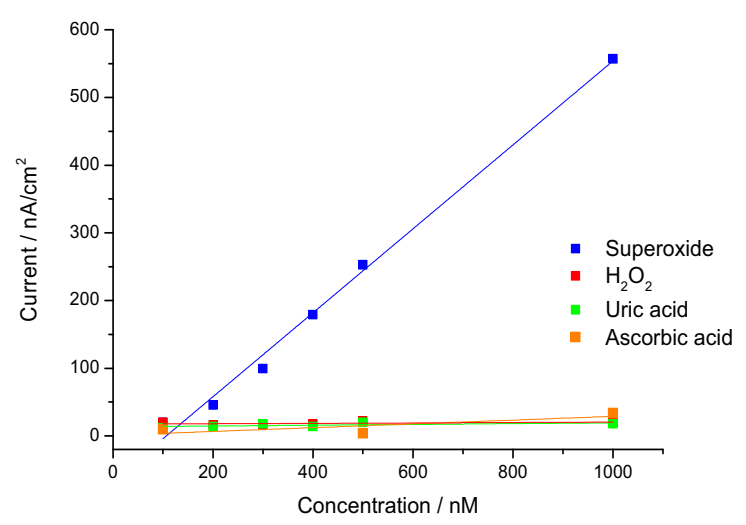

Figure 4: Calibration plot for superoxide anion and relevant interfering substances.

Adding defined aliquots of xanthine substrate to the measurement solution (PBS) containing the enzyme XOD results in a spike like production of superoxide, as all substrate is immediately converted to superoxide radical which decays fast to hydrogen peroxide and uric acid. The sensor response to this superoxide concentration spikes is shown in figure 5 whereas the peak height of a similar experiment is shown in figure 6.

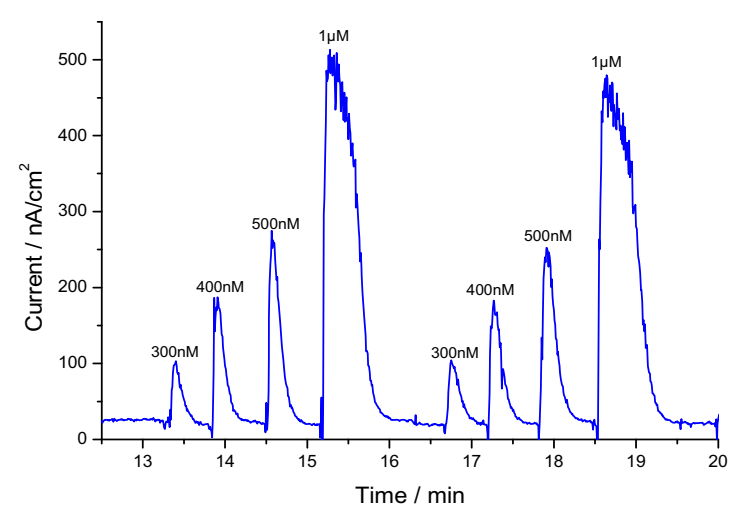

Figure 5: Amperometric sensor signal for increasing, non-continuous superoxide production. Legend numbers indicate the added xanthine concentration.

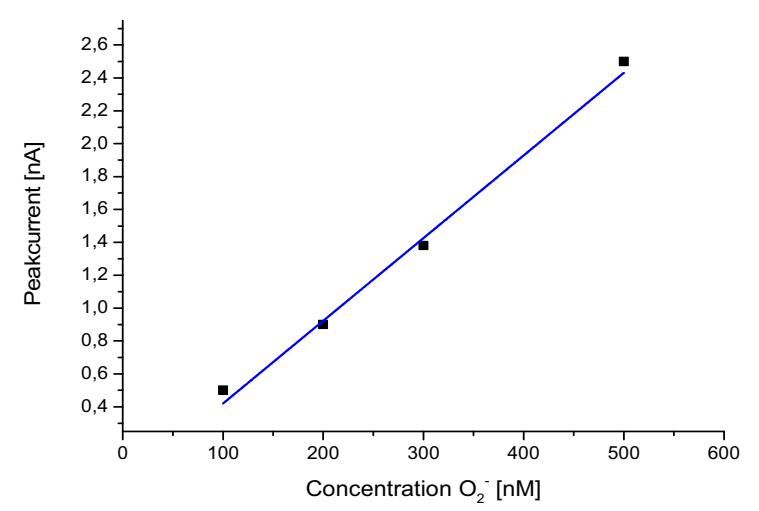

Figure 6: Resulting peak current vs. calculated superoxide concentration.

The fast superoxide decay can easily be followed by direct oxidation. As the signal reaches the baseline, no interferent contribution can be assumed in the identical concentration range.

The selectivity towards other interferents as well as the protection against electrode fouling could be enhanced by covering the electrodes with electropolymers or plasma supported polymer membrane deposition [3].

\section{Conclusion}

It was demonstrated that enzyme-less superoxide sensors show results comparable to enzyme-based approaches with a fast sensor response and sufficient selectivity against main interferents. The utilization of bare gold electrodes and biocompatible polymer membranes allows the full integration of superoxide biosensors into existing multiparameter capable cell culture monitoring system SCCF.

\section{References}

[1] P. Ebbesen, E. O. Pettersen, T. A. Gorr, G. Jobst, K. Williams, J. Kieninger, et al., "Taking advantage of tumor cell adaptations to hypoxia for developing new tumor markers and treatment strategies", Journal of Enzyme Inhibition and Medicinal Chemistry, vol. 24, 1-39 ( 2009)

[2] J. Kieninger, J. A. Sandvik, E. O. Pettersen, G. Jobst, K. Aravindalochanan, G. A. Urban, "Monitoring of Peri-Cellular Oxygen Levels in Tumor Cell Cultures by Amperometric Oxygen Sensor Array", IEEE SENSORS 2010, Waikoloa, HI, USA, 2010

[3] L. Ledernez, F. Olcaytug, G. A. Urban, H. K. Yasuda, "Magnetically Enhanced $15 \mathrm{kHz}$ Glow Discharge of Methane", Plasma Chemistry and Plasma Processing, 659-667 (2007)

[4] B. Ge, F. Lisdat, "Superoxide sensor based on cytochrome $c$ immobilized on mixed-thiol SAM with a new calibration method", Analytica Chimica Acta 454, 53-64 (2002) 
[5] Fridovich , I. and J. M. McCord, "Quantitative Aspects of Production of Superoxide Anion Radical by Milk Xanthine Oxidase“, Journal of Biological Chemistry 245(16), 4053-4059 (1970)

[6] D. Behar, G. Czapski, et al., "Acid Dissociation Constant and Decay Kinetics of Perhydroxyl Radical", Journal of Physical Chemistry 74(17), 3209-3213 (1970) 